\title{
Interpretation of quantum measurement as well as cognitive problem through the consciousness model
}

\author{
Dhananjay Pal \\ Email address: \\ dhananjay.pal123@gmail.com,paldhananjay46@yahoo.com
}

Pharmacy College, Bengal School of Technology, Sugandha-Delhi Road, Chuchura, Dist.-Hooghly, West Bengal, INDIA, PIN-712 102

\section{To cite this article:}

Dhananjay Pal. Interpretation of Quantum Measurement as Well as Cognitive Problem Through the Consciousness Model. American Journal of Physics and Applications. Vol. 1, No. 2, 2013, pp. 38-44. doi: 10.11648/j.ajpa.20130102.12

\begin{abstract}
A single field emerged at the origin of the universe, already containing within itself the blueprint of the physical universe. The primordial single field triggered the onset of the universe. Most physicists believe that a single super-force dominated the first instants of creation. Scientists have arrived at a simple but decisive conclusion that consciousness is very much a part of the universe, like other objects. Our consciousness model involving thought-carrying particle (TCP), thought retaining particle (TRP) and thought force $\left(\mathrm{T}_{\mathrm{F}}\right)$ signifies the existence of universal consciousness that exists along with the universe. This universal consciousness is a functional state of the universal mind. This universal mind (UM) is evolved at the Big Bang from void. The UM is constituted by these TCP and TRP in the inherent presence of thought force $\left(\mathrm{T}_{\mathrm{F}}\right)$. Thought force $\left(\mathrm{T}_{\mathrm{F}}\right)$ is an expression of universal consciousness. The Thought force $\left(\mathrm{T}_{\mathrm{F}}\right)$ being the primordial quantum field functions as the original super-force. $\mathrm{T}_{\mathrm{F}}$ being the original super-force functions as the origin of all the fundamental fields. TCP is the carrier of thought force $\left(\mathrm{T}_{\mathrm{F}}\right)$ that, in turn, appears to be the origin of all the fields. The quantized energy $\left(\varepsilon_{T}\right)$ of TCP is responsible to cause the universal consciousness as well as the cosmic microwave background radiation temperature. The individual consciousness owes its origin to the universal consciousness created by the same $\varepsilon_{T}$. The same $\varepsilon_{T}$ is the energy responsible for generating thought force $\left(\mathrm{T}_{\mathrm{F}}\right) . \mathrm{T}_{\mathrm{F}}$ being an expression of the universal consciousness is applicable to any inanimate object as well as to any biological system (having thinking ability). The $\mathrm{T}_{\mathrm{F}}$ exerts its functions both in vitro and in vivo. We showed the existence of thought force in microcosm [ $\mathrm{T}_{\mathrm{F}}$ (micro)] and thought force in macrocosm $\left[\mathrm{T}_{\mathrm{F}}(\right.$ macro) $]$. This $\mathrm{T}_{\mathrm{F}}$ (micro) is theoretically found to be stronger than the strong nuclear force. $T_{F}$ (macro) is theoretically found to be weaker even than the gravitational force. TCP, TRP, $T_{F}$ (micro), $T_{F}$ (macro), and the thought force $\left(\mathrm{T}_{\mathrm{F}}\right)$ in vitro and thought force $\left(\mathrm{T}_{\mathrm{F}}\right)$ in vivo play significant roles in the quantum measurement as well as cognitive problem.
\end{abstract}

Keywords: Universal Mind (UM), Thought Force (TF), TF (Micro), TF (Macro), Though-Carrying Particle (TCP), Thought Retaining Particle (TRP), Quantized Energy $\left(\varepsilon_{T}\right)$ of TCP, Cosmic Microwave Background Radiation (CMBR)

\section{Introduction}

In Eastern philosophical traditions, consciousness is intrinsic to the universe, whereas in most Western views, consciousness is extrinsic, emerging from complex computation. How can these views be reconciled?

It is most relevant and important to indicate the names of various eminent physicists like Erwin Schrödinger, Eugene Wigner, Brian Josephson, John Wheeler, Roger Penrose, Henry P Stapp, Freeman J. Dyson, Paul Davies, David Bohm, Basil Hiley, Fritjof Capra, Fred Alan Wolf and Amit Goswami who have addressed the inclusion of consciousness in their work. Consciousness is to be taken into account.
Consciousness model of $\mathrm{Pal}$ et al [1-3] involving TCP, TRP and thought force $\left(\mathrm{T}_{\mathrm{F}}\right)$ signifies the existence of universal consciousness that exists along with the universe.

Bhaumik [4] mentioned, "It would be reasonable to presume that the universe originated in a unity of all fields at or near Planck's dimension. John Wheeler strongly believes that "in defining any useful concept of reality" we have to take into account "the indispensable place of the participating observer---evidenced in quantum mechanics," the foregoing cannot be really true unless consciousness is as essential as aspect of nature as are the fields that give rise to force and matter and the primary field that gave rise to them". 
Physicists determined that underlying quantum fields give birth to elementary particles. Bhaumik [4] mentioned that Frank Wilczek pointed out, "In quantum field theory, the primary elements of reality are not individual particles, but underlying fields. Thus, for example, all electrons are but excitations of an underlying field, naturally called electric field". The same holds true for all the fundamental particles of which matter is made.

TCP cannot exist without TRP and vice versa. Many physicists believe that unifying all the forces, including gravity, into a single theory would require a phenomenon called super-symmetry. With super-symmetry, every fermion would have a boson twin, and vice-versa. TCP that behaves like boson should accompany its supersymmetrical partner TRP that functions like fermion in the generalized simpler way. Thus TCP like boson cannot have anti-particle. But TRP that functions like fermion should have its anti-particle and here it is shown as Anti-TRP. It is to be noted that these TCP and TRP function like wavicle: wave-particle duality.

1.1. In contrast to the usual linear sequence of matter, body, life, brain, mind, consciousness, here the proposed cyclic sequence is first universal consciousness (a functional state of the universal mind), and then matter, body, life, brain, and regeneration of mind and consciousness. The evolution of life with mind and consciousness is possible purely due to the inherent existence of universal consciousness which exists along with the universe. The human nervous system is evolved to provide an appropriate material structure to individualize the universal consciousness, a characteristic of reality, pervading all manifestations.

Consciousness model of Pal et al [1-3] involving TCP, TRP and thought force $\left(\mathrm{T}_{\mathrm{F}}\right)$ signifies the existence of universal consciousness that exists along with the universe. Pal et al [3] showed that this universal consciousness is a functional state of Universal Mind (UM). Pal et al [3] explained that the UM is evolved at the Big Bang from the eternal Void. This Void, in turn, is the source of infinite energy. And this UM is a finer matter. The individual mind being a constituent of the UM is also a finer matter. The constituents of the UM and individual mind are the same. The ultimate constituents of matter and mind are the same as both mind and matter are aspects of one fundamental reality, which is called UM. The brain is the mediating link or interface between the individual mind and body.

Pal et al [3] explained that the constituents of the UM are the ultimate constituents of matter itself as everything in this universe is a manifestation of this UM. Pal et al [3] expressed that the UM is constituted by these TCP and TRP in the inherent presence of thought force $\left(\mathrm{T}_{\mathrm{F}}\right)$. Pal et al $[1,3]$ further explained that the ultimate constituents of matter and mind are these TCP and TRP in the inherent presence of thought force $\left(\mathrm{T}_{\mathrm{F}}\right)$ in vitro and thought force $\left(\mathrm{T}_{\mathrm{F}}\right)$ in vivo. Pal [5] explained the existence of the thought force $\left(\mathrm{T}_{\mathrm{F}}\right)$ that, in turn, is the primordial quantum field.

1.2. Pal et al [3] developed three different equations expressing the quantized energy ( $\left.\varepsilon_{T}\right)$ of TCP. The value of
$\mathcal{E}_{T}$ in one of the three equations is shown below:

$\mathcal{E}_{T}=$

$4.384 \times 10^{-16} \mathrm{erg} \equiv 2.73 \times 10^{-4} \mathrm{eV} \equiv 2.73^{0} \mathrm{~K} \cong C M B R$ temperature $\equiv 2.725^{\circ} \mathrm{K}$

It is to be noted that $1 \mathrm{erg} \cong 0.6241807 \times 10^{12} \mathrm{eV}$ and

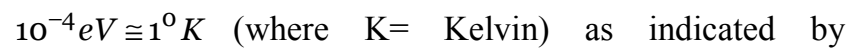
Weisskopf [6].

1.3. Pal et al [3] expressed that the CMBR temperature is due to $\varepsilon_{T}$, the quantized energy of TCP where $\varepsilon_{T}=4.384 \times 10^{-16} \mathrm{erg} \equiv 2.73^{\circ} \mathrm{K}$ that is very close to $2.725^{\circ} \mathrm{K}$ (CMBR temperature). As per Pal et al [3], it is interesting to note that all of the three different equations ultimately give rise to the same result that is equivalent to the CMBR temperature. A sort of relationship is thus observed between the $\varepsilon_{T}$ and the CMBR temperature. This coincidence is thus signifying a probable role of TCP on the maintenance of CMBR temperature. Further, this coincidence is also signifying the existence of these TCP in the presence of TRP. TCP cannot exist without TRP and vice versa. The presence of TCP, TRP and Thought force $\left(T_{F}\right)$ in the universe is thus indicated and expressed mathematically. At present we are unable to explain when and how these TCP and TRP decoupled from the primordial cosmic soup.

Pal et al $[1,3]$ showed that the quantized energy $\left(\varepsilon_{T}\right)$ of TCP is responsible to cause the universal consciousness as well as the cosmic microwave background radiation temperature. The individual consciousness owes its origin to the universal consciousness created by the same $\varepsilon_{T}$.

Ultimately this $\mathcal{E}_{T}$ represents universal consciousness. The existence of CMBR temperature indicates the existence of the TCP in the presence of TRP. The existence of TCP ensures the existence of the thought force $\left(T_{F}\right)$. Further, this $\mathrm{T}_{\mathrm{F}}$ being an expression of the quantized energy $\left(\boldsymbol{\varepsilon}_{T}\right)$ of TCP exerts its functions both in vitro and in vivo.

\section{Thought Force}

Pal [5] explained the existence of Thought force $\left(\mathrm{T}_{\mathrm{F}}\right)$. This Thought force $\left(\mathrm{T}_{\mathrm{F}}\right)$, an expression of the universal consciousness, is the primordial quantum field that, in turn, functions as the primary unified field. This $\mathrm{T}_{\mathrm{F}}$ being an expression of the universal consciousness is applicable to any inanimate object as well as to any biological system (having thinking ability). Thus the $\mathrm{T}_{\mathrm{F}}$ being an expression of the universal consciousness exerts its functions both in vitro and in vivo.

Physicists determined that underlying quantum fields give birth to elementary particles. Pal [5] expressed that the thought force $\left(\mathrm{T}_{\mathrm{F}}\right)$ is the primordial quantum field. Thought force $\left(T_{F}\right)$ being the primordial quantum field functions as the primary unified field. Thought force $\left(\mathrm{T}_{\mathrm{F}}\right)$ being the primordial quantum field gives birth to TRP that appears to be the origin of all the matter particles. TCP is the carrier of 
thought force $\left(\mathrm{T}_{\mathrm{F}}\right)$ that, in turn, appears to be the origin of all the fields. TCP thus appears to be the origin of all the field particles.

In a purpose to involve both the non-living and living systems of the world, Pal [5] has shown the existences of these TCP, TRP and thought force $\left(\mathrm{T}_{\mathrm{F}}\right)$ in vitro and thought force $\left(\mathrm{T}_{\mathrm{F}}\right)$ in vivo.

Pal (5) expressed that the non-living system of the world is governed by the thought force $\left(\mathrm{T}_{\mathrm{F}}\right)$ in vitro and this Thought force $\left(\mathrm{T}_{\mathrm{F}}\right)$ in vitro gives rise to $\mathrm{T}_{\mathrm{F}}$ (micro), SNF, EMF, WNF, GF and $\mathrm{T}_{\mathrm{F}}$ (macro)

where $\mathrm{T}_{\mathrm{F}}$ (micro) $=$ Thought force in microcosm, $\mathrm{SNF}=$ Strong nuclear force, $\mathrm{EMF}=$ Electromagnetic force, $\mathrm{WNF}=$ Weak nuclear force, $G F=$ Gravitational force and $T_{F}$ (macro) $=$ Thought force in macrocosm. It is to be noted here that $\mathrm{T}_{\mathrm{F}}$ (micro) is a stronger force than the $\mathrm{SNF}$ and $\mathrm{T}_{\mathrm{F}}$ (macro) is a weaker force even than the GF.

$\mathrm{Pal}$ (5) also expressed that the living system of the world is governed by the thought force $\left(\mathrm{T}_{\mathrm{F}}\right)$ in vivo and this Thought force $\left(\mathrm{T}_{\mathrm{F}}\right)$ in vivo is a type of force that represents the biological 'thought' which is the action of mind. This 'thought' being a type of force controls the 'thought processes' involving the firing of neurons through the quantum mechanical activities of these TCP and TRP in the presence of consciousness. Consciousness in living organisms is a process which involves the quantum mechanical activities of these TCP and TRP, the ultimate constituents of any matter as well as any mind in the inherent presence of thought force $\left(\mathrm{T}_{\mathrm{F}}\right)$ in vitro and the thought force $\left(\mathrm{T}_{\mathrm{F}}\right)$ in vivo as indicated by $\mathrm{Pal}$ [5]. These TCP and TRP govern the activities of neurons (not the other way round). Neurons are simply the equipments used to generate consciousness and awareness.

This consciousness, in turn, is the quantized energy $\left(\varepsilon_{T}\right)$ of TCP. The thought force $\left(\mathrm{T}_{\mathrm{F}}\right)$ in vivo is demonstrated in numerous experiments in which thought has an effect on a physical process (often known as mind over matter). This biological 'thought' is a type of force that can cause movement. Controlling movement through thought alone is observed in several experiments conducted by many scientists as indicated by Pal (5). These experiments thus signify the existence of thought force $\left(\mathrm{T}_{\mathrm{F}}\right)$ in vivo.

Pal [5] explained the existence of $\mathrm{T}_{\mathrm{F}}$ (micro) (= Thought force in microcosm). This $\mathrm{T}_{\mathrm{F}}$ (micro) is the strongest interaction (a new class of 'extra strong' interaction). It is stronger than SNF (Strong Nuclear Force).

Pal [5] has also shown the existence of $\mathrm{T}_{\mathrm{F}}$ (macro) (= Thought force in macrocosm). It is the "weakest force" which is much weaker even than the gravity.

\section{TCP and TRP Play the Significant Roles in the So-Called 'Non-Locality' Problem in Quantum Mechanics}

Pal et al [3] and Pal [7] explained that the universe exists along with the universal consciousness. This universal consciousness exists throughout the universe in the form of universe wide web $(u w w)$ covering fields, particles, space- time continuum, dark matter, dark energy, void and all its known and unknown parameters along with all its inhabitants (with or without consciousness). The quantized energy $\left(\varepsilon_{T}\right)$ of TCP represents universal consciousness. This universal consciousness is to be taken into account, but usually ignored.

Pal (5) expressed that Thought force $\left(T_{F}\right)$ is bridging the microcosm and macrocosm through the generation of a universal web of quantum fields of $T_{F}$ (micro) and $T_{F}$ (macro) due to the existence of the quantum mechanical activities of these TCP in the inherent presence of TRP.

Wilczek [8] expressed that intangible quantum fields fill everything in the universe, including the voids inside atoms and the universe, space between galaxies. Quantum electrodynamics has shown that even the vacuum of space, utterly devoid of matter or conventional forms of energy, actually seethes with activity. The possibility of the existence of a universal web of quantum fields signifies the existence of the $T_{F}$ (micro) and $T_{F}$ (macro) due to the existence of the quantum mechanical activities of these TCP in the inherent presence of TRP.

Pal et al [3] and Pal [7] explained that everything in this universe is interlinked and intertwined through the existence of the quantum mechanical activities of these TCP in the inherent presence of TRP. The universal consciousness functions as a universe wide web (uww) covering the universe as a whole with all its parameters (including void) and inhabitants (with or without consciousness). In this picture, uww of consciousness, quantum concepts like wave particle dualism, position momentum uncertainty, non-locality and concept of unified field become somewhat understandable as all the entities of this universe are interlinked and intertwined. In this picture, the universal consciousness replaces ether of yesteryears and contains the whole of the universe in its fold.

The 'spooky action-at-a-distance' is built in nature through the existence of these TCP and TRP in the presence of thought force $\left(\mathrm{T}_{\mathrm{F}}\right)$ in vitro and Thought force $\left(\mathrm{T}_{\mathrm{F}}\right)$ in vivo. The spooky non-local correlations-at-a-distance might evidence a Holy Ghost at work through the existence of the quantum mechanical activities of these TCP and TRP in the inherent presence of Thought force $\left(\mathrm{T}_{\mathrm{F}}\right)$ in vitro and Thought force $\left(\mathrm{T}_{\mathrm{F}}\right)$ in vivo.

\section{TCP, TRP and the Thought Force (TF) In Vitro and Thought Force (TF) In Vivo Play Significant Roles in the Quantum Measurement As Well As Cognitive Problem}

Pal et al $[1,3]$ showed that the quantized energy $\left(\varepsilon_{T}\right)$ of TCP is responsible to cause the universal consciousness as well as the cosmic microwave background radiation temperature. The individual consciousness owes its origin to the universal consciousness created by the same $\varepsilon_{T}$. Ultimately this $\mathcal{E}_{T}$ represents universal consciousness. The 
existence of CMBR temperature indicates the existence of the TCP in the presence of TRP. Pal et al $[1,3]$ explained that these TCP and TRP are the ultimate constituents of any matter as well as any mind in the inherent presence of thought force $\left(\mathrm{T}_{\mathrm{F}}\right)$ in vitro and the thought force $\left(\mathrm{T}_{\mathrm{F}}\right)$ in vivo.

Wigner [9] became interested in the Vedanta philosophy of Hinduism, particularly its ideas of the universe as an all pervading consciousness. He commented "It was not possible to formulate the laws (of quantum theory) in a fully consistent way without reference to consciousness."

Schrödinger (1935) described a thought experiment in which a cat is placed in a box into which poison is released when triggered by a particular quantum event. Schrödinger pointed out that according to the Copenhagen interpretation, the cat would be both dead and alive until the box was opened and the cat observed by a conscious human. Schrödinger cat is the first instance (Thought Experiment) to show a relation between mind (consciousness) and matter in terms of probability (uncertainty). But the present physics has not yet been able to solve the riddle of consciousness.

Wigner [10] also conceived the Wigner's friend thought experiment in physics, which is an extension of the Schrödinger's cat thought experiment. The Wigner's friend experiment asks the question: "At what stage does a 'measurement' take place?" Wigner designed the experiment to highlight how he believed that consciousness is necessary to the quantum-mechanical measurement processes.

\section{Significance of TCP and TRP in Quantum Measurement As Expressed By Pal Et $A l$ (2)}

The philosophical foundation of quantum mechanics is based on the duality concept of complementary notions indicated by Dutta [11], particles and waves, discrete and continuous, localized and non-localized characteristics of quantum behaviour expressed through the equations for energy and momentum as $E=h v$ and $p=h \lambda^{-1}$, respectively. These equations fundamentally signify the qualitative aspect of quantum mechanics, because the quantitative aspect, involving the quantum measurement problem, is governed by the uncertainty relations that restrict the simultaneous accurate determination of position and momentum, energy and time etc. The uncertainty or imprecision in the measured values is an inherent feature of the subatomic world. In our view, this subatomic world is controlled by the quantum mechanical activities of these TCP and TRP with which any matter as well as any mind is constituted. And the functional state of mind is consciousness which, in turn, plays the most prominent role in the quantum measurement. Thus the quantum measurement involving the role of consciousness is governed by these TCP, TRP, $\mathrm{T}_{\mathrm{F}}$ (micro), $\mathrm{T}_{\mathrm{F}}$ (macro), and the thought force $\left(\mathrm{T}_{\mathrm{F}}\right)$ in vitro and thought force $\left(\mathrm{T}_{\mathrm{F}}\right)$ in vivo. It is to be noted that these TCP and TRP function like wavicle: wave-particle duality.

Schrödinger [12] expressed: "We cannot make any factual statement about a given natural object (or physical system) without 'getting in touch' with it. This 'touch' is a real physical interaction. Even if it consists only in our 'looking at the object' the latter must be hit by light-rays and reflect them into the eye, or into some instrument of observation. This means that the object is affected by our observation. You cannot obtain any knowledge about an object while leaving it strictly isolated. The theory goes on to assert that this disturbance is neither irrelevant nor completely survey-able. Thus after any number of painstaking observations the object is left in a state of which some features (the last observed) are known, but others (those interfered with by the last observation) are not known, or not accurately known. This state of affair is offered as an explanation why no complete, gap-less description of any physical object is ever possible".

As argued by many authors, "observation" itself plays a very important role in quantum measurements due to its interdependence with phenomena. The very act of "observation" distorts the state under 'observation' through the projection of TCP. The expected consequence is the "apparent" non-determinism in quantum measurements. The concept of "observation-phenomenon interdependence" is entirely governed by the quantum mechanical activities of these TCP in the presence of TRP. These TCP in the presence of TRP not only obey the uncertainty principle but also involve two different velocities (viz. V, the mechanical motion of the TCP and $\mathrm{u}$, the propagation of the associated wave when they are related by $\mathrm{u}=\mathrm{c}^{2} / \mathrm{V}$ ) present at a time. These TCP in the presence of TRP behave, in general, like photons or bio-photons, yet they may be mathematically allotted a mass $\left(\mathrm{m}_{\mathrm{T}}\right)$ equivalent to $5.5 \times 10^{-37} \mathrm{~g}$ or $3.09 \times 10^{-}$ ${ }^{13} \mathrm{GeV}$. The validity of Heisenberg's uncertainty principle concerning the apparent duality of "certainty" and "uncertainty" will always prevail in this space-time continuum so long there is the question of 'physical observation' through the quantum mechanical activities of these TCP (in the presence of TRP), of course, in the inherent presence of consciousness. Thus Heisenberg's uncertainty principle is a fundamental inescapable property of this physical universe. The nature normally does not permit us to surpass this apparent duality in the network of "space-time-causation" by the way of physical observation. Quantum physics has given hopes of unveiling the mysteries concerning the laws of consciousness. It has accomplished the very important task of explaining the photon activity in the central nervous system, which begins from receiving the information signals. The chemical forces, which control the interaction of atoms and molecules inside the microtubles of the brain during the intermediate stages of processing consciousness, and transfer signals from one neuron to another across the synaptic clefts, are indeed quantum mechanical activities of these TCP and TRP, the ultimate constituents of matter and mind. The consciousness itself is functioning as an inter-linking agent between the animate and inanimate through the quantum mechanical activities of these TCP and TRP in the inherent presence of Thought force $\left(\mathrm{T}_{\mathrm{F}}\right)$ in vitro and Thought force $\left(\mathrm{T}_{\mathrm{F}}\right)$ in vivo as indicated by Pal et al [1-3]. 


\section{What Is the Science Behind the Recognition of An Observable Object in the Field of Cognitive Science? TCP and TRP Play Significant Roles in the Field of Cognitive Neuroscience As It Is Expressed by Pal Et $\boldsymbol{A l}$ (2)}

The explanation which the present science gives in regard to the 'process of seeing' only explains the formation of a picture on the retina of the physical eye through the activity of brain, but gives no explanation whatever how these pictures come to the consciousness. It is known that nearly 40 areas of the brain are ultimately involved with the overall function of visual perception. And vision is only one component of awareness. There are others like sounds, aroma, emotions etc. They are all occurring at the same moment. Structurally, a full grown human brain is a highly efficient electromagnetic system of a mass of nervous tissues consisting of more than 10 billion cells weighing up to $2 \%$ of the body's weight, but consuming more than $25 \%$ of the system's total energy bill. The brain caps this excess energy mainly for structuring consciousness. The foremost role of the brain is to receive external information through various channels, to differentiate them bit by bit and spread them throughout the brain in waves of electrical pulsation for symbolization. The symbolized information is maintained for future recognition of any object through the integration into consciousness. It is here that judgment plays a role, comparing present information with past experience, the data of which is kept coded in the memory shelves. Judgment, which is an important component of consciousness, regulates activity to make necessary adjustments with the surroundings for survival.

The mind of animate must create the wave towards any sensation in order to perceive it. In every phenomenon in nature we contribute at least half share and the nature brings half as indicated by Vivekananda [13]. If our half share is taken off, the thing must stop to exist for us. Nobody knows what is external; when we try to know it, it has to become that material which we furnish by projecting the mind (consisting of TCP and TRP) in the presence of consciousness. The reflections formed in the physical eye (through stimuli) only serve to call the attention of the conscious mind to the objects of its perception. Perception is a faculty by which mind learns to know what is going on within itself. To see a thing is to perceive the existence of its appearance within one's own mind. To see a thing is identical with touching it with the mind. The individual mind of a man being one with the Universal mind extends through space; and the things themselves that exist within the periphery of our mind can be perceived through the consciousness, the apparent center of which is thought to be located in the brain. If the mind touches an object through the quantum mechanical activities of these TCP and TRP, the impressions have to travel all the way to the brain. What we know of the external is as it is moulded, formed, fashioned by our own mind, which, in turn, exerts its actions through the brain.

The reigning tenet of quantum mechanics is the uncertainty principle. A consequence of the uncertainty principle is that the presence of an observer or experimenter determines the outcome of the observation or experiment. Simply stated, this means there is no objective reality; you 'create' what you see through the quantum mechanical activities of these TCP and TRP in the presence of consciousness. The quantized energy $\left(\varepsilon_{T}\right)$ of TCP represents universal consciousness. The individual consciousness owes its origin to the universal consciousness created by the same $\varepsilon_{T}$. Pal et al $[1,3]$ explained that these TCP and TRP are the ultimate constituents of any matter as well as any mind in the inherent presence of thought force $\left(\mathrm{T}_{\mathrm{F}}\right)$ in vitro and the thought force $\left(\mathrm{T}_{\mathrm{F}}\right)$ in vivo.

It is to be noted that TCP cannot exist without TRP and vice versa. These TCP and TRP function like wavicle: wave-particle duality. Both the TCP and TRP are interchangeable in the presence of consciousness, of course,

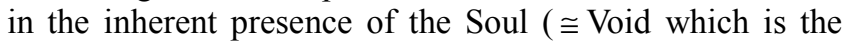
source of infinite energy) to complete the process of observation in order to see the observable 'object'.

There is 'something' which is outside, which is only the occasion, the suggestion; and upon that 'suggestion' I project the TCP of my mind with the help of consciousness to see (identify) this external 'suggestive something'.

What is the process of observation? How do we see? It is to be noted that these TCP and TRP are interchangeable in the presence of consciousness to complete the process of observation in order to see the observable 'object'. When I project the TCP of my mind with the help of consciousness to see the external observable 'object', this TCP from my mind is converted into TRP in the presence of my consciousness. This TRP cannot exist without TCP. These TRP and TCP thus 'create' the observable object that I see.

TCP from this observable 'object' is to be returned to me in order to complete the process of observation for seeing the observable 'object'. This returned TCP from the object is to be converted into TRP in the presence of my consciousness and this converted TRP is to be retained and stored within my mind for future use. This conversion of TCP from the observable 'object' into TRP within my mind is necessary for forming an impression of the object within my mind so that I can retain that image with the help of TRP within my mind for future 'recognition' when I would come across the same 'thing'. This is possible through the power of the Soul ( $\cong$ Void which is the source of infinite energy) in the presence of consciousness.

It is to be noted that these TCP and TRP are the ultimate constituents of any matter as well as any mind in the inherent presence of thought force $\left(\mathrm{T}_{\mathrm{F}}\right)$ in vitro and the thought force $\left(\mathrm{T}_{\mathrm{F}}\right)$ in vivo. It is to be understood that when we try to see an observable external 'object', it has to become that material which we furnish by projecting the mind (consisting of TCP and TRP) in the presence of consciousness and it takes the form that I see.

Thus, there is no objective reality; I 'create' what I see 
through the quantum mechanical activities of these TCP and TRP in the presence of my consciousness. These TCP and TRP are interchangeable in the presence of consciousness to 'create' and then see the observable 'object'. "Matter is an expression of mind, not separate from mind, but mind manifested materially".

Consciousness, not matter, is the ground of all existence, declares University of Oregon physicist Goswami through his published (1993) book, "The Self-Aware Universe: How Consciousness Creates the Material World". He accepts the Vedantic view and holds that the universe is self-aware, and that consciousness creates the physical world. Matter is an expression of mind, not separate from mind, but mind manifested materially.

John Wheeler strongly believed that "in defining any useful concept of reality" we have to take into account "the indispensable place of the participating observer--evidenced in quantum mechanics" as indicated by Bhaumik [4]. Wheeler has speculated that reality is created by observers in the universe. His time-bending notion is that our "observership brings the universe into being".

Wheeler indicated that the process of recognizing an observable object is really a type of participation on the part of the observer for a specific object to be observed. This process of recognizing an observable object is possible through the projection of mind of the observer in the presence of his / her consciousness.

How could an object's existence depend upon the act of observation? This is due to the fact that an object's existence as well as the act of observation through the prevailing consciousness is totally dependent on the existence of the quantum mechanical activities of these TCP and TRP in the presence of consciousness. The quantized energy $\left(\varepsilon_{T}\right)$ of TCP represents universal consciousness. The individual consciousness owes its origin to the universal consciousness created by the same $\varepsilon_{T}$. Pal et al $[1,3]$ explained that these TCP and TRP are the ultimate constituents of any matter as well as any mind in the inherent presence of thought force $\left(\mathrm{T}_{\mathrm{F}}\right)$ in vitro and the thought force $\left(\mathrm{T}_{\mathrm{F}}\right)$ in vivo.

How do we all see the same things? Because we all have similar parts (i.e. TCP and TRP) of the Universal Mind that, in turn, is constituted by these TCP and TRP. Those who have like minds will see like things, and those who have not, will not see alike. I will never know that external 'something' in the absence of the function of my mind ( $\cong$ consciousness) through which the soul ( $\cong$ Void), the master ruler of the living body, exerts its functions. Thus both the external something and the internal Soul are always remaining unknown and unknowable throughout. Their presence can be visualized only in the presence of the function of mind generating consciousness. So, there is always a bridge between the internal world and the external world through the vehicle of mind (composed these TCP and TRP) in the presence of consciousness.

In our view, the brain is only a fine "instrument" through which the mind exerts its functions with the help of TCP and TRP. The "binding problem" associated with the concepts of coherence can also be tackled through the quantum mechanical activities of these TCP and TRP. A single unified experience of an object having different

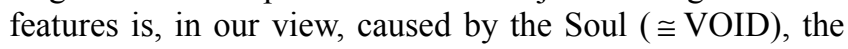
master ruler of the body. And this Soul, with the help of consciousness, exerts its functions through the finer instrument called "mind". Being guided by the Soul, the mind can, with the help of TCP and TRP, correlate and assemble all the different stimuli (relating to the different features of an object) into a unified experience of a single thing through the utilization of the instrumental brain. The modern scientists are not at all ready, at least theoretically,

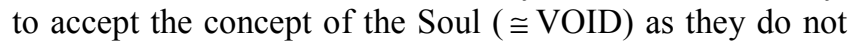
have even today authentic scientific knowledge relating to the physics as well as chemistry of this Soul. Yet the "science of observation" in the field of "cognitive neuroscience" can be explained through the existence of Soul and mind in the inherent presence of consciousness. In the presence of consciousness, these TCP and TRP are inter-convertible to carry and retain a specific "thought" and also for its communication from one person to another.

\section{Discussion and Conclusion}

\subsection{Discussion}

The quantized energy $\left(\varepsilon_{T}\right)$ of TCP represents universal consciousness. Consciousness is created and maintained by the TCP in the inherent presence of TRP. Thus consciousness involves the quantum mechanical activities of these TCP and TRP to which Heisenberg's uncertainty principle is equally applicable. Consciousness will have to play its own role in quantum measurements; and quantum measurements are thus liable to be governed by the uncertainty relations. The term "observation" is nothing but the utilization and involvement of the consciousness itself indicated by Pal et al [2].

Bhaumik [4] pointed out, "In quantum physical experiments, an observer's consciousness is capable of bringing about a particular outcome from the coexisting possibilities inherent in any quantum system".

Pal et al $[1,3]$ explained, "It is possible that the quantum enigma can be explained by accepting the existence of the quantum mechanical activities of these TCP and TRP that are the ultimate constituents of any matter as well as any individual mind, the functional state of which, in turn, generates 'consciousness'. This 'consciousness' is acting as an inter-linking agent between the animate and inanimate".

Samanta-Laughton [14] expressed, "At a quantum level particles can exist in many states at a time. What brings them out of this superposition is a matter of debate, but according to the Copenhagen interpretation, it is the act of observing a particle that determines what it is. Bohr went further and said that there is no objective reality 'out there'. Things only exist when we observe them. This implies that the whole universe exists only in our consciousness. Although controversial, the Copenhagen interpretation has stood the test of time with its bizarre philosophical implications".

Schrödinger [12] expressed, "It is the same elements that 
go to compose my mind and the world. This situation is the same for every mind and its world; in spite of the unfathomable abundance of 'cross-references' between them. The world is given to me only once, not one existing and one perceived. Subject and object are only one. The barrier between them cannot be said to have broken down as a result of recent experience in the physical sciences, for this barrier does not exist".

Pal [5] explained the testability for the existence of TCP and TRP along with the Thought force $\left(\mathrm{T}_{\mathrm{F}}\right)$. Pal [5] also mentioned several experimental results to signify the functional existence of quantum mechanical activities of these TCP and TRP, the ultimate constituents of matter and mind in the inherent presence of thought force $\left(\mathrm{T}_{\mathrm{F}}\right)$ in vitro and the thought force $\left(\mathrm{T}_{\mathrm{F}}\right)$ in vivo.

\subsection{Conclusion}

Role of TCP, TRP and the thought force $\left(\mathrm{T}_{\mathrm{F}}\right)$ in vitro and thought force $\left(\mathrm{T}_{\mathrm{F}}\right)$ in vivo is shown here to play significant roles in the quantum measurement as well as cognitive problem.

It appears that the standard model of physics is to be correctly tuned by ascertaining the constituents of quarks and leptons in the presence of unified field in order to form a possible gross bridge between present physics and cognitive science, psychology and natural sciences.

\section{References}

[1] D. Pal and A.U. De, Physics of consciousness and its model may provide guidelines to solve many scientific problems. Neuroquantology 1, 17-28 (2004)

[2] D. Pal, and A.U. De, Consciousness model: Significance of thought-carrying particles and thought-retaining particles in quantum measurement as well as cognitive problem. NeuroQuantology 2, 115-116 (2005)

[3] D. Pal, and A.U. De, The cosmic microwave background radiation temperature signifying the existence of the thought-carrying particle, thought retaining particle and thought force. NeuroQuantology 10: Issue3; 428-442 (September 2012)

[4] M. Bhaumik, Code Name GOD. (Penguin Books India Pvt. Ltd., 11 Community Centre, Panchsheel Park, New Delhi 110 017, India) pp. 132-133; 161-162; 167; 171; 177; 183; 185- 186; 89-190; 198 (2006)

[5] D. Pal, Existence of thought force and its characteristics. Communicated to American Journal of Modern Physics (AJMP) (2013)

[6] V. F. Weisskopf, The Origin of the Universe, The World of Physics, (Simon and Schuster, 1230 Avenue of Americas, New York 10020) 3, pp. 10 (1987)

[7] D. Pal, Existence of universal consciousness and its characteristics. Accepted for publication in 2014 International Conference on Advanced Education and Management (ICAEM2014) Beijing, China (2013)

[8] F. Wilczek, Mass Without Mass I: Most of matter. Physics Today 11 (November, 1999)

[9] E. Wigner, Symmetries and Reflections: Scientific Essays. (MIT Press) (1970)

[10] E. Wigner, "Remarks on the Mind-Body Problem", in The Scientist Speculates, I. J. Good, ed. pp. 284-302, Heinemann, London (1961); Basic Books, New York (1962). Reprinted in Wigner (1967/1983)

[11] SK. Dutta, The Noninfinite, Nonzero Quantized Energy Limits and Their Physical Significance, Physics Essays 8:469 (1995)

[12] E. Schrödinger, What Is Life? With Mind and Matter, First published in 1944. (Cambridge University Press) 125-127 (1967)

[13] S. Vivekananda, Meditation. The complete works of Swami Vivekananda. (Advaita Ashrama, 5, Dehi Entally Road Cal7000014) 4: 229 (1989)

[14] M. Samanta-Laughton, A Beginner's Guide to Consciousness. The Naked Scientists: Science Radi \& Science Podcasts 2005 